\title{
BMJ Open Hyperacute stroke thrombolysis via telemedicine: a multicentre study of performance, safety and clinical efficacy
}

\author{
Nicholas Richard Evans (D) , ${ }^{1,2}$ Lynda Sibson, ${ }^{3}$ Diana J Day, ${ }^{3}$ Smriti Agarwal, ${ }^{3}$ \\ Raj Shekhar, ${ }^{4}$ Elizabeth A Warburton ${ }^{2}$
}

To cite: Evans NR, Sibson L, Day DJ, et al. Hyperacute stroke thrombolysis via telemedicine: a multicentre study of performance, safety and clinical efficacy. BMJ Open 2022;12:e057372. doi:10.1136/ bmjopen-2021-057372

- Prepublication history for this paper is available online. To view these files, please visit the journal online (http://dx.doi. org/10.1136/bmjopen-2021057372).

Received 13 September 2021 Accepted 10 December 2021

Check for updates

(c) Author(s) (or their employer(s)) 2022. Re-use permitted under CC BY-NC. No commercial re-use. See rights and permissions. Published by BMJ.

${ }^{1}$ Department of Medicine, University of Cambridge, Cambridge, UK

${ }^{2}$ Department of Clinical Neurosciences, University of Cambridge, Cambridge, UK ${ }^{3}$ Department of Stroke Medicine, Cambridge University Hospitals NHS Foundation Trust, Cambridge, UK

${ }^{4}$ Department of Stroke Medicine, Queen Elizabeth Hospital King's Lynn NHS Foundation Trust, King's Lynn, UK

Correspondence to Dr Nicholas Richard Evans; ne214@cam.ac.uk

\section{ABSTRACT}

Objectives Timely thrombolysis of ischaemic stroke improves functional recovery, yet its delivery nationally is challenging due to shortages in the stroke specialist workforce and large geographical areas. One solution is remote stroke specialist input to regional centres via telemedicine. This study evaluates the usage and key metrics of performance of the East of England Stroke Telemedicine Partnership - the largest telestroke service in the UK-in providing hyperacute stroke care.

Design Prospective observational study.

Setting The East of England Stroke Telemedicine Partnership provides a horizontal 'hubless' model of out-of-hours hyperacute stroke care to a population of 6.2 million across a 7500 square mile semirural region. Participants All (2709) telestroke consultations between 1 January 2014 and 31 December 2019.

Main outcome measures Thrombolysis decision, pre-thrombolysis and post-thrombolysis stroke severity (National Institutes of Health Stroke Scale, NIHSS), haemorrhagic complications, and hyperacute pathway timings.

Results Over the period, 1149 (42.4\%) individuals were thrombolysed. Thrombolysis rates increased from 147/379 (38.8\%) in 2014 to 225/490 (45.9\%) in 2019. Median (IQR) pre-thrombolysis NIHSS was $10(6-17)$, reducing to $6(2-14)$ 24-hour post-thrombolysis $(p<0.001)$. Post-thrombolysis haemorrhage occurred in 27 cases (2.3\%). Over the period, median (IQR) door-to-needle time reduced from 85 (65-108) min to 68 (55-97.5) $\min (p<0.01)$, driven by improved imaging-to-needle times from 52.5 (38-72.25) min to 42 $(30.5-62.5) \min (p<0.01)$. However, the same period saw an increase in median onset-to-hospital arrival time from 77.5 (60-109.25) min to 95 (70-135) min $(p<0.001)$.

Conclusions The results from this large hyperacute telestroke cohort indicate two important points for clinical practice. First, telemedicine via a hubless horizontal model provides a clinically effective and safe method for delivering hyperacute stroke thrombolysis. Second, improved door-to-needle times were offset by a concerning rise in prehospital timings. These findings indicate that although telemedicine may benefit in-hospital hyperacute stroke care, improvements across the whole stroke pathway are essential.

\section{INTRODUCTION}

Thrombolysis is a mainstay in the management of hyperacute ischaemic stroke, and

\section{Strengths and limitations of this study}

This study of a large cohort of individuals undergoing hyperacute stroke assessment via telemedicine provides robust evidence for the efficacy and safety of a horizontal telestroke network across large semirural populations in the UK.

- The study also demonstrates a concerning sustained trend of rising onset-to-hospital arrival times that represents an important target for quality improvement to improve acute stroke care.

- The study has implications for stroke service design to reduce the inequalities of stroke services given the ongoing challenges of shortages in the specialist stroke workforce and the large geographical distances seen in parts of the UK.

- This large observational study describes trends in service use and performance against key Stroke metrics in one of the UK's largest and most established telestroke networks, though direct comparison against in-person thrombolysis services is outside the scope of the study.

recognition that its timely administration is associated with better functional outcomes has led to the principle that 'time is brain'. In-hospital timings from arrival to neuroimaging to thrombolysis bolus remain key metrics of performance, and the recognition that centres performing more thrombolysis have faster door-to-needle times with no differences in safety outcomes has underpinned the argument for the centralisation of stroke services. $^{2}$

The East of England is a semirural region covering $19000 \mathrm{~km}^{2}$ with a population of 6.2 million that typifies two major challenges encountered globally in the delivery of thrombolysis: geography and workforce. Although centralisation of stroke services may improve performance in urban areas, in rural settings, the increased travel time to a centralised neuroscience centre (estimated to be $45-60 \mathrm{~min}$ in our region) may offset any reduction in hospital arrival to treatment 
time. However, the provision of hyperacute reperfusion therapies in smaller local centres is frequently limited by a shortage of stroke specialists, particularly out of hours, leading to heterogeneous stroke services both geographically and temporally. The shortage of stroke physicians in the UK, where $40 \%$ of stroke units have at least one unfilled consultant post, ${ }^{3}$ means that it is not feasible to have an on-site stroke specialist presence at all times. Such challenges have been exacerbated during the increased service pressures caused by the COVID-19 pandemic.

This combination of geographical and workforce challenges has necessitated alternative approaches to deliver hyperacute stroke care. Telemedicine-or 'telestroke' in the setting of stroke care-has been advocated as a potential solution for providing regional centres with stroke specialist advice remotely. ${ }^{4}$ Different models of telestroke networks have developed: the 'hub-and-spoke' modelthe most common model where stroke specialists at a central hub provide advice to regional spokes either prior to local treatment or transfer to the hub-or the less common horizontal network; a 'hubless' model where teleconsultations are performed by stroke specialists from the centres within the network. ${ }^{5}$

The East of England Stroke Telemedicine Partnership follows this latter model. Since its inception in 2010, it has developed into the UK's largest and longest-running telestroke network; comprising seven acute hospitals with hyperacute stroke units (listed below) and staffed by 12 stroke specialists from all centres in the region to provide out-of-hours hyperacute stroke care.

To date, most evaluation of telestroke networks has considered hyperacute care in hub-and-spoke models and frequently focused exclusively on in-hospital performance metrics. This study evaluates trends in service utilisation, rates of thrombolysis, complications and performance metrics (including in the context of the whole hyperacute pathway) in the hubless model used in the East of England Stroke Telemedicine Partnership.

\section{METHODS}

\section{Design of the telestroke network}

The East of England Stroke Telemedicine Partnership is hosted by Cambridge University Hospitals NHS Foundation Trust and supported by a Telemedicine Manager and Technical Manager. It provides hyperacute stroke care overnight (17:00-08:00) on weekdays, Friday 17:00 to Monday 08:00 over weekends, and 24-hour coverage on bank holidays. Following a successful pilot phase that demonstrated early feasibility and increased rates of thrombolysis across the region, ${ }^{6}$ the network has grown to comprise seven acute hospitals with hyperacute stroke units: Ipswich Hospital (East Suffolk and North Essex NHS Foundation Trust), James Paget University Hospital (James Paget University Hospitals NHS Foundation Trust), Lister Hospital (East and North Hertfordshire NHS Trust), Peterborough City Hospital (North West Anglia NHS Foundation Trust), Watford General
Hospital (West Hertfordshire Hospitals NHS Trust) and the West Suffolk Hospital (West Suffolk NHS Foundation Trust). An eighth hospital (Princess Alexandra Hospital) was also included prior to the closure of its hyperacute stroke unit.

\section{Patient eligibility}

Individuals were eligible for telestroke consultation if they fulfilled eligibility for hyperacute reperfusion therapy according to standard clinical guidelines. ${ }^{7}$ For the purposes of analysing timings of the complete hyperacute stroke pathway (from onset to thrombolysis bolus), only individuals who had out-of-hospital strokes with complete data sets were included in the analysis.

\section{Data collection}

Data were collected prospectively for all telestroke consultations between 1 January 2014 and 31 December 2019. Consultant stroke physicians completed a standardised audit form for all telestroke consultations prospectively at the time of the encounter. This secure online audit form was introduced in 2014 and includes demographic data (age and sex), clinical data (National Institutes of Health Stroke Scale, NIHSS), hyperacute pathway timing (times of onset, hospital 'door' arrival, time of neuroimaging with CT and time of alteplase bolus delivery). Intervals were taken as the difference in minutes between these events, with door-to-needle and CT-to-needle, representing the time between either hospital arrival or CT scan and administration of the thrombolysis bolus ('needle'), respectively. Further follow-up data (24-hour repeat NIHSS, post-thrombolysis haemorrhage) and any missing data from the audit forms were completed retrospectively with data from the Sentinel Stroke National Audit Programme. All data were collected as part of routine clinical care and were anonymised at time of collection.

\section{Telestroke technology}

The emergency department of each participating centre has a telemedicine cart comprised of a personal computer in a bespoke mobile trolley (Parity Medical, Wirral, UK) with an additional high-resolution camera mounted on top of the screen. Remote consultant stroke physicians have access to a dedicated 17" laptop coupled to the telemedicine cart using Visionable (Visionable, London, UK) videoconferencing software that allows rapid and secure access to the virtual consultation. Verbal informed consent is obtained from the patient prior to the teleconsultation, and teleconsultations are not recorded (either audio or video). Imaging performed at the centre is shared with the consultant stroke physician using Image Exchange Portal (Sectra Linköping, Sweden), with radiographers at the centre transferring it via urgent transfer protocols. Images were interpreted by the on-call telestroke stroke physician and subsequently formally reported by local radiology services. 


\section{Statistical analysis}

Continuous data were assessed for normality using the Shapiro-Wilk method, with parametric data reported as mean (SD) and non-parametric data reported as median (IQR). Comparison of two groups was performed by the Student's t test (for parametric data) or the Wilcoxon Rank Sum test (for non-parametric data). Comparison of prethrombolysis and post-thrombolysis NIHSS was performed using an equivalent paired test. Although comparisons between 2014 and 2019 data used the aforementioned tests for comparing two groups, differences between year-by-year data of the study period were tested using one-way analysis of variance(ANOVA) (parametric data) or Kruskal-Wallis (non-parametric) methodology. Correlations between two groups of continuous data used Pearson methodology for parametric data or Spearman's methodology $\left(r_{s}\right)$ for non-parametric data. Data were analysed using R (V.3.6.1, 2019, R Foundation for Statistical Computing, Vienna, Austria).

\section{Approvals}

Verbal consent was obtained from the patient or next of kin prior to thrombolysis as per standard clinical care. The registry of anonymised (non-patient identifiable) consultations on the East of England Stroke Telemedicine Partnership is maintained for service evaluation and quality improvement with data governance approvals for analysis and reporting from each participating centre. All stroke clinicians in the partnership hold an honorary contract with Cambridge University Hospitals NHS Foundation Trust. This project was approved by Cambridge University Hospitals NHS Foundation Trust (PRN9187).

\section{Data sharing}

Anonymised data are available on reasonable request from the corresponding author.

\section{Role of the funding source}

No specific funding was received for this study. The East of England Stroke Telemedicine Partnership itself is funded for clinical service provision at a cost of $£ 63000$ per annum to each participating centre. Data collection was performed routinely for clinical governance as described above. The design of the study, data collection, data analysis and writing of the manuscript were performed without involvement from NHS funding bodies. The corresponding author (NRE) had full access to all the data in the study and had final responsibility for the decision to submit for publication.

\section{Patient and public involvement}

The design of the East of England Stroke Telemedicine Partnership, in particular the patient-facing aspects, has been influenced by the experiences of the individuals treated by it, with the refinements introduced in response to their (and their families') feedback being integral to developing the service. The study objectives and design were informed by these experiences, and wider consultation with stroke survivors and the public on priorities for stroke care within the region. As intervention was delivered as standard clinical care, there was no additional burden of intervention from a research perspective. Findings from this manuscript will be disseminated via newsletter, and link to the manuscript on the Partnership website and social media.

\section{RESULTS}

\section{Patient cohort and service usage}

Over the 6-year period, there were 2709 telestroke consultations, of which 1149 (42.4\%) were thrombolysed. Of the 1560 consultations where the decision was made not to thrombolyse, 342 were diagnosed as stroke mimics at the time of consultation. The patient characteristics are shown in table 1 . The number of telestroke consultations and the proportion receiving thrombolysis increased over the study period, rising from $147 / 379(38.8 \%)$ in 2014 to 225/490 (45.9\%) in 2019 (figure 1).

Over the whole period, the median duration of the telestroke consultation for thrombolysed cases was 20 (14-30) min. There was a downward trend in consultation duration (median 23 (14-39) min in 2014 vs 19 (13-24.25) $\mathrm{min}$ in 2019, $\mathrm{p}<0.01$ for comparison between these 2 years, $\mathrm{p}<0.001$ over the whole study period) (table 2).

In 2019, the East of England Stroke Telemedicine Partnership accounted for 225 (42.1\%) of the total number of thrombolysed cases across the seven centres of the telestroke network (535 cases in total across both in and out-of-hours).

\section{Clinical outcomes}

Median prethrombolysis NIHSS was 10 (6-17), falling to $6(2-14)$ at 24-hours post-thrombolysis $(\mathrm{p}<0.001)$.

Complications occurred in 30 cases (2.6\%): 20 (1.7\%) with any intracranial bleeding (either symptomatic or asymptomatic), 7 (0.6\%) with extracranial bleeding and $3(0.3 \%)$ with anaphylactic reactions.

\section{Hyperacute pathway timings}

Year-by-year consultation duration and hyperacute pathway timings are shown in table 2. The reduction in door-to-needle times (from a median of 85 (65-108) $\min$ in 2014 to $68(55-97.5) \mathrm{min}$ in $2019, \mathrm{p}<0.01)$ was secondary to a significant reduction in median CT-toneedle times from (52.5 (38-72.25) $\mathrm{min}$ in 2014 to 42 $(30.5-62.5) \mathrm{min}$ in $2019, \mathrm{p}<0.01)$ but not in median doorto-CT times from (30 (19.75-42) $\mathrm{min}$ in 2014 vs 27 (18.536.5) $\min$ in 2019, $\mathrm{p}=0.19$ ) over the period.

Prealerting the stroke physician immediately following triage on arrival in the emergency department (typically prealerted by the Stroke Specialist Nurse reviewing suspected stroke calls on their arrival in the emergency department, either as ambulance transfers or selfattendances) had a significant effect on reducing median CT-to-needle times (42.5 (28.25-61) $\mathrm{min}$ if prealerted vs 47.5 (35-66) $\min$ if not, $\mathrm{p}<0.01)$. 
Table 1 Patient characteristics for thrombolysed and non-thrombolysed (both all presentations and stroke only presentations) cohorts

\begin{tabular}{lcllll}
\hline & & \multicolumn{2}{l}{ Not thrombolysed } & & \\
\cline { 2 - 6 } & & $\begin{array}{l}\text { Not } \\
\text { thrombolysed- }\end{array}$ & $\begin{array}{l}\text { Significance } \\
\text { (against } \\
\text { thrombolysed) }\end{array}$ & $\begin{array}{l}\text { Not } \\
\text { thrombolysed- } \\
\text { strokes only }\end{array}$ & $\begin{array}{l}\text { Significance } \\
\text { (against } \\
\text { thrombolysed) }\end{array}$ \\
\hline Number & 1149 & 1560 & & 1218 & \\
\hline Median age (IQR) & $75(64-83)$ & $73(56.25-84)$ & $\mathrm{p}<0.01$ & $76(63-85)$ & $\mathrm{p}=0.26$ \\
\hline Number of men (\%) & $622(54.1 \%)$ & $732(46.9 \%)$ & $\mathrm{p}<0.001$ & $586(48.1 \%)$ & $\mathrm{p}<0.01$ \\
\hline Hypertension (\%) & $314(27.3 \%)$ & $340(21.8 \%)$ & $\mathrm{p}<0.001$ & $288(23.6 \%)$ & $\mathrm{p}<0.01$ \\
\hline Atrial fibrillation (\%) & $116(10.1 \%)$ & $163(10.4 \%)$ & $\mathrm{p}=0.77$ & $150(12.3 \%)$ & $\mathrm{p}=0.04$ \\
\hline Diabetes mellitus (\%) & $99(8.6 \%)$ & $102(6.5 \%)$ & $\mathrm{p}=0.04$ & $83(6.8 \%)$ & $\mathrm{p}=0.10$ \\
\hline Median NIHSS (IQR) & $10(6-17)$ & $5(2-11)$ & $\mathrm{p}<0.001$ & $5(2-12)$ & $\mathrm{p}<0.001$ \\
\hline Median consult duration (IQR) & $20(14-30)$ & $15(9-26)$ & $\mathrm{p}<0.001$ & $15(8-25)$ & $\mathrm{p}<0.001$ \\
\hline Median onset-door (IQR) & $90(66-124)$ & $110(71-180)$ & $\mathrm{p}<0.001$ & $115(74-189.25)$ & $\mathrm{p}<0.001$ \\
\hline Median door-CT (IQR) & $27(17-38)$ & $35(23-55)$ & $\mathrm{p}<0.001$ & $34(22-55)$ & $\mathrm{p}<0.001$ \\
\hline
\end{tabular}

NIHSS, National Institutes of Health Stroke Scale.

Although in-hospital door-to-needle times reduced over the period, the prehospital times increased significantly, with median symptom onset-to-door times increasing from $77.5(60-109.25)$ min to 95 (70-135) min between 2014 and 2019, respectively $(\mathrm{p}<0.001)$. Consequently, the total symptom onset-to-needle time did not change significantly during the study period (table 2 ).

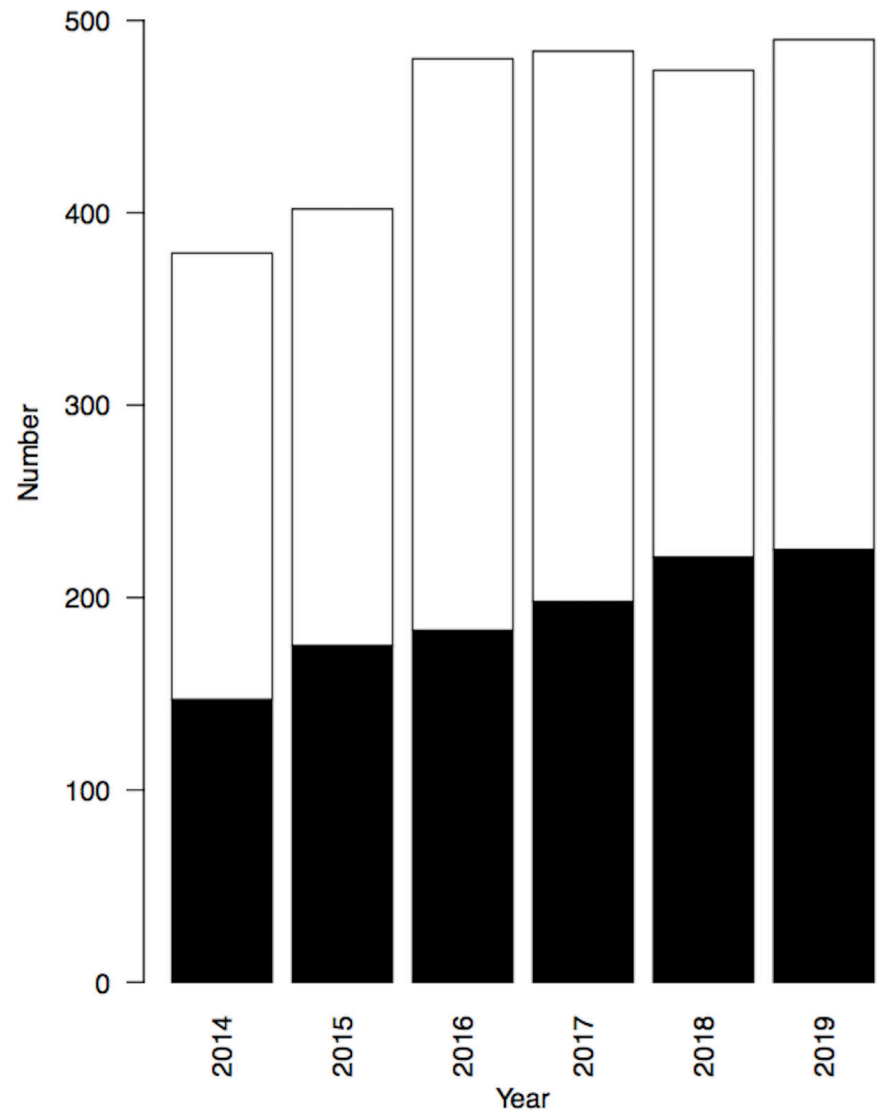

Figure 1 Number of teleconsultations by year. Black=thrombolysed, white=not thrombolysed.
There was a very weak negative association between the onset-to-door time and door-to-needle time $\left(\mathrm{r}_{\mathrm{s}}=-0.10\right.$, $\mathrm{p}<0.001)$. There was also a very weak positive association between the door-to-CT and CT-to-needle times $\left(\mathrm{r}_{\mathrm{s}}=0.10\right.$, $\mathrm{p}<0.01)$.

There was a very weak association between NIHSS and door-to-CT time $\left(\mathrm{r}_{\mathrm{s}}=-0.11, \mathrm{p}<0.01\right)$. There was no significant relationship between NIHSS and CT-to-needle $\left(r_{s}=0.02, p=0.66\right)$ or overall door-to-needle time $\left(r_{s}=-0.04\right.$, $\mathrm{p}=0.27)$. There was no correlation between onset-toneedle times and 24-hour NIHSS $\left(\mathrm{r}_{\mathrm{s}}=0.01, \mathrm{p}=0.70\right)$, though there was a very weak association with the change in NIHSS $\left(r_{s}=0.10, p<0.01\right)$ following thrombolysis.

\section{Prehospital timings}

Prehospital timings were available for 128 patients seen during the second half of 2019. The median symptom onset-to-emergency call time was 30.5 (14-61.5) min, median call-to-arrival time was 21.5 (11.75-31) min, median time on scene was 24 (19-31.25) min and median scene-to-hospital arrival time was 16 (10-24.25) min. There was a weak negative association between NIHSS and the call-to-arrival time $\left(\mathrm{r}_{\mathrm{s}}=-0.20, \mathrm{p}=0.04\right)$, and the association between NIHSS and onset-to-call time narrowly missed significance $\left(r_{s}=-0.19, p=0.06\right)$. However, there was no association between NIHSS and either time spent on scene $\left(\mathrm{r}_{\mathrm{s}}=0.03, \mathrm{p}=0.74\right)$ or scene-to-hospital arrival time $\left(\mathrm{r}_{\mathrm{s}}=-0.07, \mathrm{p}=0.43\right)$.

\section{DISCUSSION}

In this analysis of the UK's largest and longest-running telestroke network, we report two important findings: first, the use of a horizontal telestroke network to deliver hyperacute stroke thrombolysis is feasible and clinically effective, with comparable safety to thrombolysis delivered by stroke specialists in person. Second, although 
Table 2 Performance of the telestroke service for thrombolysed cases by year

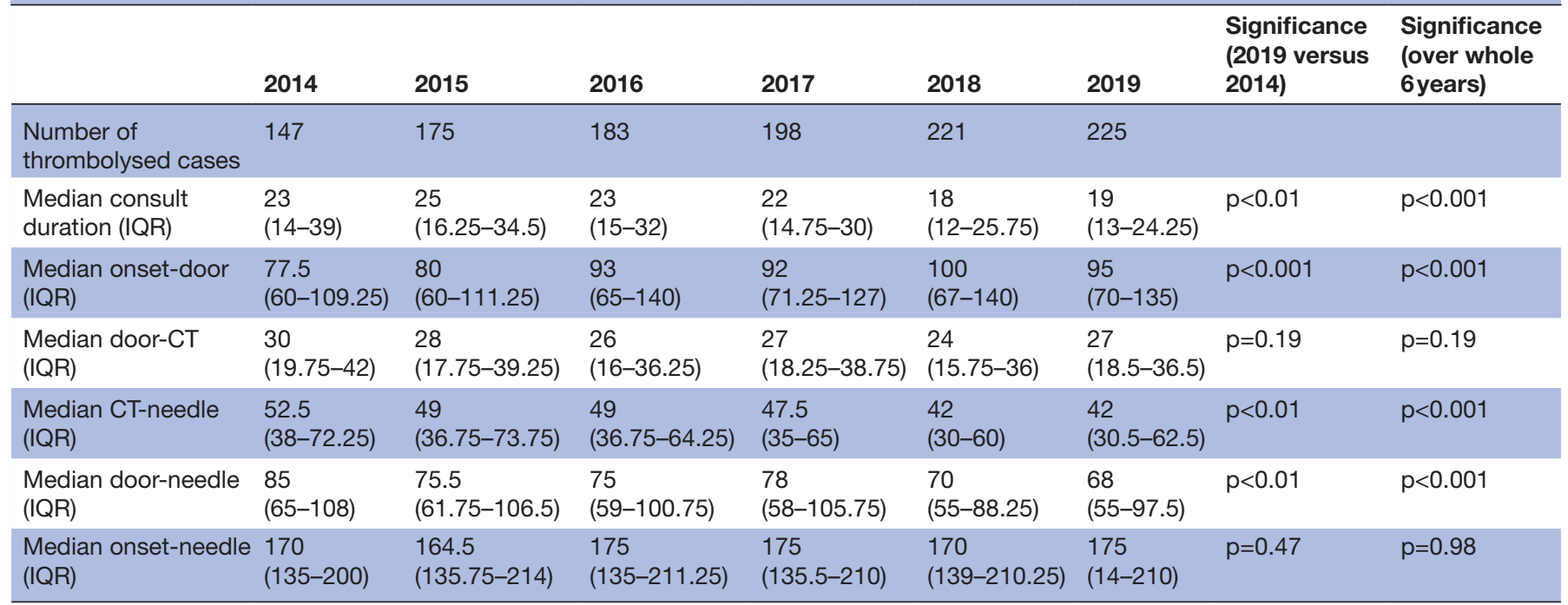

All times are in minutes. Significance is shown as direct comparison of 2014 and 2019 data, and over the whole 6-year period.

our service has seen improved in-hospital times, there is a concerning sustained rise in prehospital timings for stroke.

The horizontal network model of telemedicinedelivered hyperacute care has several advantages over the hub-and-spoke model encountered more commonly. The pooled resource of stroke clinicians benefits sustainability of the service in contrast to staffing from a single centre and also fosters a sense of shared ownership of the partnership that benefits cross-site collaboration. However, there have been relatively few studies evaluating horizontal networks. A small study in Finland reported that half of those thrombolysed had a favourable outcome (modified Rankin Scale (mRS) 0-2), outcomes comparable to the local neuroscience centre. ${ }^{8}$ Prehospital times were longer and in-hospital times were shorter for the academic centre compared with the telestroke network, but the overall onset-to-needle times were the same for both. ${ }^{9}$ Our study supports the efficacy of such a network in the UK and adds to the body of evidence with a large cohort over a sustained period.

Prior to the introduction of the East of England Stroke Telemedicine Partnership, only 2\% of acute strokes admitted across participating hospitals in 2009 received thrombolysis. The rise in the proportion of acute stroke thrombolysed is multifactorial, but the Stroke Telemedicine Partnership has been a major factor in increasing the accessibility to stroke specialist expertise that would not be feasible with centres acting independently.

Thrombolysis in our cohort was associated with a significant reduction in NIHSS in the first 24 hours. This is consistent with reports that thrombolysis has been associated with a greater percentage change in NIHSS at 24 hours compared with those treated with placebo. ${ }^{10}$ Although we did not collect longer-term outcomes, such early neurological change has been found to be associated with improved 90-day clinical outcomes. ${ }^{11} 12$
Telestroke-guided thrombolysis in our horizontal network was not associated with an increased complication rate, falling at the lower end of the recognised rate of post-thrombolysis symptomatic intracerebral haemorrhage of $2 \%-7 \% .{ }^{13}$ Similar findings have been observed in a meta-analysis of telemedicine-guided thrombolysis versus delivery in a stroke centre, where there was no difference in symptomatic intracerebral haemorrhage rates, functional independence or mortality. ${ }^{14}$

The improvement in door-to-needle time appears to be driven by the significant reduction in CT-to-needle time. This likely represents a learning effect as individual centres have become accustomed to the hyperacute pathway and technology as well as improved clinical decision-making, as suggested by the significant decrease in the duration of the telestroke consultation over the evaluation period. Furthermore, our data suggest that pragmatic approaches, such as the effect of prealerting the telestroke consultant, have an important role in reducing this clinical decision-making time by eliminating avoidable delays. This is consistent with findings that an interval between arrival and telestroke alert of more than 15 min has been found to be associated with an eightfold increase in the likelihood a door-to-bolus time longer than an hour. ${ }^{15}$

This learning effect is particularly true for the stroke specialist nurses co-ordinating care in the emergency department and for the consultant stroke physicians responding to the calls. In contrast, this learning effect is likely to be less pronounced among the on-site physicians, typically specialist trainees in general medicine specialties, due to frequent rotations and is unlikely to be contributing to the sustained improvement in performance over the duration of the study. Our study aimed to evaluate the performance of our horizontal network and its evolution over a decade, and as such did not compare the performance against out-of-hours timings in 
on-site consultations by a stroke specialist (in part due to the fundamental issue of the shortage of stroke specialists for out-of-hours in-person assessments at district general hospitals across the region), though increasingly in-person and telestroke-delivered thrombolysis are reporting no significant difference in door-to-needle times, ${ }^{16}$ or even faster door-to-needle times than historical in-person controls in rural hospitals. ${ }^{17}$

The median door-to-needle time seen in our network remained longer than the target of an hour advocated by many quality improvement initiatives. ${ }^{18} 19$ However, these timings were similar to those reported over a corresponding period in hub-and-spoke telestroke networks in Victoria (Australia) and Boston (USA), both reporting a median door-to-needle time of $73 \mathrm{~min} .{ }^{172}$ Other horizontal networks, such as the Kaiser Permanente Northern California and the Telemedical Project for Integrative Stroke Care (TEMPiS) networks, have reported median door-to-needle times of $34 \mathrm{~min}$ and $39 \mathrm{~min}$, respectively. ${ }^{921}$ However, it is important to note that they are not directly comparable to our network: the TEMPiS network operates $24 / 7$ with a number of centres having either an in-hour or 24/7 neurology presence, while the Kaiser Permanente Northern California network used predominantly emergency physicians with previous experience of thrombolysis prior to introduction of the telestroke network. In contrast, thrombolysis in our service is managed by non-stroke specialist doctors, typically specialist registrars (residents) on-call for general medicine in the district general hospitals and operates solely out-of-hours. It has been reported that timings in the hyperacute pathway are slower out-of-hours for all thrombolysed cases ${ }^{22}$ as well as those specifically within a telestroke network. ${ }^{23}$ Hence, longer door-to-needle times seen in our network may reflect differences in when the service operates and who is staffing it in-person and need to be considered in the context of how to provide out-ofhours care when on-site specialist services are not available. However, there remains ongoing work to decrease our out-of-hours door-to-needle times further.

The second important finding is that, although the in-hospital door-to-needle time has improved over this period, this improvement is countered by a rise in prehospital timings. Essentially, the near-20 min reduction in door-to-needle times was offset by the approximate $20 \mathrm{~min}$ increase in symptom onset-to-hospital arrival times. This trend of rising prehospital times in hyperacute stroke care is increasingly recognised at a national level in the $\mathrm{UK}^{24}$ as well as internationally. ${ }^{20}$ This consistent trend in rising prehospital timings in our data may be due to several reasons, including the rise in demand on acute ambulance services, longer time spent assessing patients on scene or delays by individuals contacting emergency services. There is also the possibility that faster in-hospital processes now mean that individuals presenting later may be able to be treated within the time window. However, the very weak negative association between onset-to-hospital arrival and in-hospital door-to-needle times suggests that this is not a major influence. Our data on prehospital timings are limited in its ability to delineate these factors and does not include the geographical distances covered by the ambulance from call-to-arrival or scene-to-hospital that may influence response times. However, it is important to reiterate that ambulances in our partnership currently attend the nearest district general hospital. Consequently, the observed increase in prehospital timings is unlikely to represent increased conveyance times when diverting to a larger comprehensive stroke centre further away. Our prehospital data suggest that stroke severity is associated, although weakly, with faster times to call emergency services and narrowly missed significance with faster response times for ambulance arrivals, though severity was neither associated with any effect on time on scene nor speed of subsequent conveyance to hospital. This may be because once a hyperacute stroke is recognised, then conveyance to the nearest hospital is treated as an emergency transfer, regardless of severity. This raises the possibility that it may be the initial recognition and assessment of stroke on the scene that will benefit most from improved detection and expedited care of stroke, which is consistent with similar reported findings. ${ }^{24}$ These prehospital timings are recorded and monitored robustly in routine clinical care, and a larger sample needs to be analysed within our partnership in order to elucidate which step in the pathway is contributing to the increasing prehospital timings, and consequently represent a target for support and intervention to reverse this trend.

This is important, as prehospital care of hyperacute strokes is a vital consideration for improving timely delivery of reperfusion therapies as well as patient selection for transfer to thrombectomy centres in cases of suspected large vessel occlusion strokes. Telemedicine has been shown to have a potential role by improving and expediting such prehospital decision-making in hyperacute stroke care by enabling remote review by stroke specialists. $^{25} 26$

The use of telemedicine to deliver thrombolysis has been shown to be cost-effective in a hub-and-spoke model when considering the long-term outcomes on stroke, particularly if shared across hospitals in the network. ${ }^{27-29}$ Furthermore, telestroke has been applied across the whole stroke pathway, including clinic follow-up ${ }^{30}$ and rehabilitation, though evidence remains limited due to study design and size. ${ }^{31}$ Given the concerning increase in onset to hospital presentation times seen here and elsewhere, there may be a role for the use of telemedicine for stroke in the prehospital setting in order to facilitate early decision-making and management.

It is important to note some limitations in our study. The diagnosis of stroke was made on clinical grounds at the time of presentation, and individuals did not routinely undergo MRI to confirm the diagnosis of ischaemic stroke. Our primary endpoint was a change in NIHSS in the first 24 hours, and further measures of clinical outcomes at a latter time point, such as mRS at 3 months, would be 
advantageous for evaluating the longer term efficacy of the service and represent important future work.

Overall, our findings demonstrate the clinical effectiveness and safety of telemedicine to facilitate hyperacute ischaemic stroke care through a 'hubless' horizontal telestroke network, providing a potential solution to the challenges of delivering thrombolysis across large geographical regions with a limited specialist stroke workforce.

Acknowledgements The authors wish to thank all members in the East of England Stroke Telemedicine Partnership for their efforts in delivering the service.

Contributors NRE conceived the design of the work, curated data, performed statistical analysis, interpreted data and wrote the first draft of the manuscript. LS collected and curated data. LS, DJD, RS, EAW developed the East of England Stroke Telemedicine Partnership. LS, DJD, SA, RS, EAW all aided data interpretation and revised the manuscript for important intellectual content. All authors approved the version to be published, and all authors agree to be accountable for all aspects of the work in ensuring that questions related to the accuracy or integrity of any part of the work are appropriately investigated and resolved. As guarantor, NRE accepts full responsibility for the work and/or the conduct of the study, had access to the data, and controlled the decision to publish.

Funding NRE is supported by an Academic Clinical Lectureship from the National Institute for Health Research (award number is not applicable).

Competing interests None declared.

Patient and public involvement Patients and/or the public were involved in the design, or conduct, or reporting, or dissemination plans of this research. Refer to the Methods section for further details.

Patient consent for publication Not applicable.

Ethics approval This project was approved by Cambridge University Hospitals NHS Foundation Trust (PRN9187).

Provenance and peer review Not commissioned; externally peer reviewed.

Data availability statement Data are available upon reasonable request. Anonymised data are available upon reasonable request from the corresponding author.

Open access This is an open access article distributed in accordance with the Creative Commons Attribution Non Commercial (CC BY-NC 4.0) license, which permits others to distribute, remix, adapt, build upon this work non-commercially, and license their derivative works on different terms, provided the original work is properly cited, appropriate credit is given, any changes made indicated, and the use is non-commercial. See: http://creativecommons.org/licenses/by-nc/4.0/.

ORCID iD

Nicholas Richard Evans http://orcid.org/0000-0002-7640-4701

\section{REFERENCES}

1 Meretoja A, Keshtkaran M, Saver JL, et al. Stroke thrombolysis: save a minute, save a day. Stroke 2014;45:1053-8.

2 Bray BD, Campbell J, Cloud GC, et al. Bigger, faster? Associations between hospital thrombolysis volume and speed of thrombolysis administration in acute ischemic stroke. Stroke 2013;44:3129-35.

3 British Association of Stroke Physicians. Meeting the future consultant workforce challenges: stroke medicine, 2019.

4 Levine SR, Gorman M. "Telestroke" : the application of telemedicine for stroke. Stroke 1999;30:464-9.

5 Hubert GJ, Santo G, Vanhooren G, et al. Recommendations on telestroke in Europe. Eur Stroke J 2019;4:101-9.

6 Agarwal S, Day DJ, Sibson L, et al. Thrombolysis delivery by a regional telestroke network--experience from the U.K. National Health Service. J Am Heart Assoc 2014;3:e000408.

7 National Institute for Health and Care Excellence. Stroke and transient ischaemic attack in over 16s: diagnosis and initial management (NICE guideline [NG128. National Institute for Health and Care Excellence, 2019.
8 Sairanen T, Soinila S, Nikkanen M, et al. Two years of Finnish Telestroke: thrombolysis at spokes equal to that at the hub. Neurology 2011;76:1145-52.

9 Hubert GJ, Meretoja A, Audebert HJ, et al. Stroke thrombolysis in a centralized and a decentralized system (Helsinki and telemedical project for integrative stroke care network). Stroke 2016:47:2999-3004.

10 Agarwal S, Scher E, Lord A, et al. Redefined measure of early neurological improvement shows treatment benefit of alteplase over placebo. Stroke 2020;51:1226-30.

11 Heitsch L, Ibanez L, Carrera C, et al. Early neurological change after ischemic stroke is associated with 90-day outcome. Stroke 2021;52:132-41.

12 Agarwal S, Cutting S, Grory BM, et al. Redefining early neurological improvement after reperfusion therapy in stroke. J Stroke Cerebrovasc Dis 2020;29:104526.

13 Yaghi S, Willey JZ, Cucchiara B, et al. Treatment and outcome of hemorrhagic transformation after intravenous alteplase in acute ischemic stroke: a scientific statement for healthcare professionals from the American heart Association/American stroke association. Stroke 2017;48:e343-61.

14 Kepplinger J, Barlinn K, Deckert S, et al. Safety and efficacy of thrombolysis in Telestroke: a systematic review and meta-analysis. Neurology 2016;87:1344-51.

15 Jagolino-Cole AL, Bozorgui S, Ankrom CM, et al. Variability and delay in Telestroke physician alert among spokes in a Telestroke network: a need for metric benchmarks. J Stroke Cerebrovasc Dis 2019;28:104332.

16 Sood R, Annoni J-M, Humm AM, et al. Distance neurological supervision using Telestroke does not increase Door-to-Needle time in acute ischemic stroke management: the experience of two regional stroke units. Front Neurol 2021;12:616620.

17 Bladin CF, Kim J, Bagot KL, et al. Improving acute stroke care in regional hospitals: clinical evaluation of the Victorian stroke telemedicine program. Med J Aust 2020;212:371-7.

18 Fonarow GC, Zhao X, Smith EE, et al. Door-to-needle times for tissue plasminogen activator administration and clinical outcomes in acute ischemic stroke before and after a quality improvement initiative. JAMA 2014;311:1632-40.

19 Hasnain MG, Paul CL, Attia JR, et al. Door-to-needle time for thrombolysis: a secondary analysis of the tips cluster randomised controlled trial. BMJ Open 2019;9:e032482.

20 Sharma R, Zachrison KS, Viswanathan A, et al. Trends in Telestroke care delivery: a 15-year experience of an academic hub and its network of spokes. Circ Cardiovasc Qual Outcomes 2020;13:e005903.

21 Nguyen-Huynh MN, Klingman JG, Avins AL, et al. Novel Telestroke program improves thrombolysis for acute stroke across 21 hospitals of an integrated healthcare system. Stroke 2018;49:133-9.

22 Campbell JTP, Bray BD, Hoffman AM, et al. The effect of out of hours presentation with acute stroke on processes of care and outcomes: analysis of data from the stroke improvement national audit programme (SINAP). PLoS One 2014;9:e87946.

23 Asaithambi G, Castle AL, Tipps ME, et al. Weekday versus weekend presentation in the acute management of ischemic stroke through telemedicine. Neurohospitalist 2020;10:115-7.

24 Haworth D, McClelland G. Call to hospital times for suspected stroke patients in the North East of England: a service evaluation. $\mathrm{Br}$ Paramed J 2019;4:31-6.

25 Mazya MV, Berglund A, Ahmed N, et al. Implementation of a prehospital stroke triage system using symptom severity and Teleconsultation in the Stockholm stroke triage study. JAMA Neurol 2020;77:691-9.

26 Geisler F, Kunz A, Winter B, et al. Telemedicine in prehospital acute stroke care. J Am Heart Assoc 2019;8:e011729.

27 Nelson RE, Saltzman GM, Skalabrin EJ, et al. The cost-effectiveness of telestroke in the treatment of acute ischemic stroke. Neurology 2011;77:1590-8.

28 Switzer JA, Demaerschalk BM, Xie J, et al. Cost-Effectiveness of hub-and-spoke Telestroke networks for the management of acute ischemic stroke from the hospitals' perspectives. Circ Cardiovasc Qual Outcomes 2013;6:18-26.

29 Demaerschalk BM, Switzer JA, Xie J, et al. Cost utility of hub-andspoke Telestroke networks from societal perspective. Am J Manag Care 2013;19:976-85.

30 Walsh J, Markus HS. Telemedicine for follow-up of rare neurological disease. Stroke 2019;50:750-3.

31 Laver KE, Adey-Wakeling Z, Crotty M, et al. Telerehabilitation services for stroke. Cochrane Database Syst Rev 2020;1:Cd010255. 